CRYSTALLOGRAPHIC COMMUNICATIONS

ISSN 2056-9890

Received 1 April 2016

Accepted 7 April 2016

Edited by M. Weil, Vienna University of Technology, Austria

Keywords: crystal structure; 1,2-cyclohexanediamine; tetrachloridozincate chloride double salt; chromium(III) complex; hydrogen bonding; synchrotron radiation.

CCDC reference: 1472901

Supporting information: this article has supporting information at journals.iucr.org/e

\section{Crystal structure of tris(trans-1,2-cyclohexane- diamine- $\left.\kappa^{2} N, N^{\prime}\right)$ chromium(III) tetrachloridozincate chloride trihydrate from synchrotron data}

\author{
Dohyun Moon ${ }^{\mathrm{a}}$ and Jong-Ha Choi ${ }^{\mathrm{b} *}$
}

${ }^{\mathbf{a} P o h a n g}$ Accelerator Laboratory, POSTECH, Pohang 37673, Republic of Korea, and ${ }^{\mathbf{b}}$ Department of Chemistry, Andong National University, Andong 36729, Republic of Korea. *Correspondence e-mail: jhchoi@anu.ac.kr

The structure of the title double salt, $\left[\mathrm{Cr}(\mathrm{rac}-\mathrm{chxn})_{3}\right]\left[\mathrm{ZnCl}_{4}\right] \mathrm{Cl} \cdot 3 \mathrm{H}_{2} \mathrm{O}(\mathrm{chxn}$ is trans-1,2-cyclohexanediamine; $\mathrm{C}_{6} \mathrm{H}_{14} \mathrm{~N}_{2}$ ), has been determined from synchrotron data. The $\mathrm{Cr}^{\mathrm{III}}$ ion is coordinated by six $\mathrm{N}$ atoms of three chelating chxn ligands, displaying a slightly distorted octahedral coordination environment. The distorted tetrahedral $\left[\mathrm{ZnCl}_{4}\right]^{2-}$ anion, the isolated $\mathrm{Cl}^{-}$anion and three lattice water molecules remain outside the coordination sphere. The $\mathrm{Cr}-\mathrm{N}(\mathrm{chxn})$ bond lengths are in a narrow range between 2.0737 (12) and 2.0928 (12) $\AA$; the mean $\mathrm{N}-\mathrm{Cr}-\mathrm{N}$ bite angle is $82.1(4)^{\circ}$. The crystal packing is stabilized by hydrogen-bonding interactions between the amino groups of the chxn ligands and the water molecules as donor groups, and $\mathrm{O}$ atoms of the water molecules, chloride anions and $\mathrm{Cl}$ atoms of the $\left[\mathrm{ZnCl}_{4}\right]^{2-}$ anions as acceptor groups, leading to the formation of a three-dimensional network. The $\left[\mathrm{ZnCl}_{4}\right]^{2-}$ anion is disordered over two sets of sites with an occupancy ratio of 0.94:0.06.

\section{Chemical context}

trans-1,2-Cyclohexanediamine (chxn) can coordinate to a central metal ion as a bidentate ligand via the two nitrogen atoms, forming a five-membered chelate ring. The synthetic procedures, crystal structures and detailed spectroscopic properties of such $\left[\mathrm{Cr}(\operatorname{chxn})_{3}\right]^{3+}$ complexes with chloride or nitrate anions have been reported previously (Morooka et al., 1992; Choi, 1994; Kalf et al., 2002). Since counter-anionic species play a very important role in coordination chemistry and supramolecular chemistry (Fabbrizzi \& Poggi, 2013; Santos-Figueroa et al., 2013), changing the type of anion can also result in different structural properties. With respect to the tetrachloridozincate anion, $\left[\mathrm{ZnCl}_{4}\right]^{2-}$, the crystal structures of complexes with trivalent chromium have been determined for $\left[\mathrm{Cr}\left(\mathrm{NH}_{3}\right)_{6}\right]\left[\mathrm{ZnCl}_{4}\right] \mathrm{Cl}$ (Clegg, 1976), $\left[\mathrm{Cr}(\mathrm{en})_{3}\right]\left[\mathrm{ZnCl}_{4}\right] \mathrm{Cl}$ (en is ethylenediamine; Pons et al., 1988) and trans- $\left[\mathrm{Cr}\left(\mathrm{NH}_{3}\right)_{2}(\right.$ cyclam $\left.)\right]\left[\mathrm{ZnCl}_{4}\right] \mathrm{Cl} \cdot \mathrm{H}_{2} \mathrm{O} \quad$ (cyclam is 1,4,8,11-tetraazacyclotetradecane; Moon \& Choi, 2016). However, a combination of the $\left[\mathrm{Cr}(\operatorname{chxn})_{3}\right]^{3+}$ cation with $\left[\mathrm{ZnCl}_{4}\right]^{2-}$ and $\mathrm{Cl}^{-}$as anions is unreported. In order to confirm that the resulting structure belongs to a double salt with $\left[\mathrm{ZnCl}_{4}\right]^{2-}$ and $\mathrm{Cl}^{-}$anions and does not contain a $\left[\mathrm{ZnCl}_{5}\right]^{3-}$ anion, we prepared this material and report here on the molecular and crystal structure of $\left[\mathrm{Cr}(\text { rac-chxn })_{3}\right]\left[\mathrm{ZnCl}_{4}\right] \mathrm{Cl} \cdot-$ $3 \mathrm{H}_{2} \mathrm{O}$, (I). 


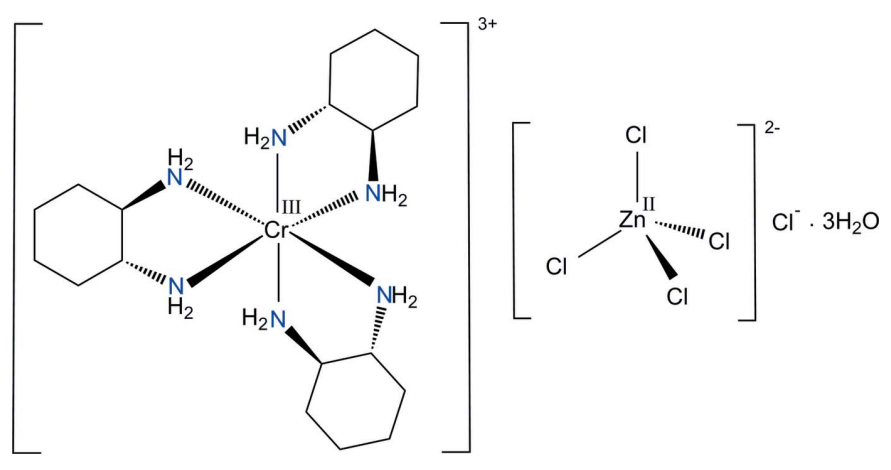

\section{Structural commentary}

First of all we performed a single-crystal structure analysis of the starting complex $\left[\mathrm{Cr}(\mathrm{chxn})_{3}\right] \mathrm{Cl}_{3} \cdot 2 \mathrm{H}_{2} \mathrm{O}$ with $98 \mathrm{~K}$ synchrotron data to determine the exact composition and coordination geometry of the $\mathrm{Cr}^{\mathrm{III}}$ ion. The complex crystallizes in the space group $I \overline{4} 2 d$ with eight formula units in a cell of dimensions $a=18.893$ (3) and $c=14.069$ (3) $\AA$. The $\mathrm{Cr}-$ $\mathrm{N}(\mathrm{chxn})$ bond lengths are in the range $2.0723(19)$ to 2.0937 (19) $\AA$, and the $\mathrm{N}-\mathrm{Cr}-\mathrm{N}$ bite angles are in the range 82.53 (7) to $82.69(10)^{\circ}$. In comparison with the bond lengths and angles of the structure of this complex determined with $223 \mathrm{~K}$ data (Kalf et al., 2002), there are no remarkable differences, and also the the crystal packing has virtually identical features.

Fig. 1 shows the molecular components of the title compound, (I), which consists of a discrete complex cation $\left[\mathrm{Cr}(\text { rac-chxn })_{3}\right]^{3+}$, three lattice water molecules, together with one tetrahedral $\left[\mathrm{ZnCl}_{4}\right]^{2-}$ and one isolated $\mathrm{Cl}^{-}$counter-ion. The nitrogen atoms of the three 1,2-cyclohexanediamine ligands define a distorted octahedral coordination environment around the $\mathrm{Cr}(\mathrm{III})$ ion with a mean $\mathrm{N}-\mathrm{Cr}-\mathrm{N}$ bite angle of $82.1(4)^{\circ}$. The resulting five-membered chelate rings of chxn ligands have the expected stable gauche conformation. The $\mathrm{Cr}-\mathrm{N}(\mathrm{chxn})$ bond lengths are in the range 2.0737 (12) to 2.0928 (12) $\AA$, in good agreement with those determined in

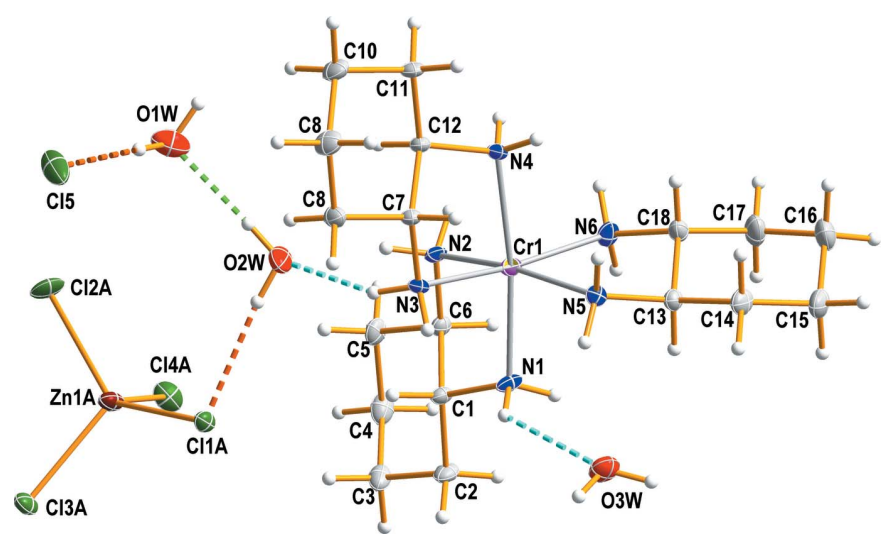

Figure 1

The structures of the molecular components of the title double salt, drawn with displacement parameters at the $50 \%$ probability level. Dashed lines represent hydrogen-bonding interactions.
Table 1

Hydrogen-bond geometry $\left(\AA,^{\circ}\right)$.

\begin{tabular}{|c|c|c|c|c|}
\hline$D-\mathrm{H} \cdots A$ & $D-\mathrm{H}$ & $\mathrm{H} \cdots A$ & $D \cdots A$ & $D-\mathrm{H} \cdots A$ \\
\hline $\mathrm{N} 1-\mathrm{H} 1 A \cdots \mathrm{Cl} 5^{\mathrm{i}}$ & 0.91 & 2.40 & $3.2535(15)$ & 157 \\
\hline $\mathrm{N} 1-\mathrm{H} 1 B \cdots \mathrm{O} 3 W$ & 0.91 & 2.36 & $3.0178(16)$ & 129 \\
\hline $\mathrm{N} 2-\mathrm{H} 2 A \cdots \mathrm{O} 2 W$ & 0.91 & 2.01 & $2.9051(17)$ & 166 \\
\hline $\mathrm{N} 2-\mathrm{H} 2 B \cdots \mathrm{Cl} 2 A^{\mathrm{ii}}$ & 0.91 & 2.45 & $3.2197(14)$ & 142 \\
\hline $\mathrm{N} 2-\mathrm{H} 2 B \cdots \mathrm{Cl} 3 B^{\mathrm{ii}}$ & 0.91 & 2.36 & $3.180(18)$ & 150 \\
\hline $\mathrm{N} 3-\mathrm{H} 3 A \cdots \mathrm{O} 2 W$ & 0.91 & 2.13 & $2.9832(16)$ & 156 \\
\hline $\mathrm{N} 3-\mathrm{H} 3 B \cdots \mathrm{Cl} 1 A^{\mathrm{iii}}$ & 0.91 & 2.52 & $3.2574(13)$ & 138 \\
\hline $\mathrm{N} 3-\mathrm{H} 3 B \cdots \mathrm{Cl} 3 A^{\mathrm{iii}}$ & 0.91 & 2.77 & $3.4547(16)$ & 133 \\
\hline $\mathrm{N} 3-\mathrm{H} 3 B \cdots \mathrm{Cl} 2 B^{\mathrm{iii}}$ & 0.91 & 2.67 & $3.471(10)$ & 147 \\
\hline $\mathrm{N} 3-\mathrm{H} 3 B \cdots \mathrm{Cl} 4 B^{\mathrm{iii}}$ & 0.91 & 2.68 & $3.35(2)$ & 131 \\
\hline $\mathrm{N} 4-\mathrm{H} 4 A \cdots \mathrm{Cl} 1 B^{\mathrm{iv}}$ & 0.91 & 2.74 & $3.473(11)$ & 138 \\
\hline $\mathrm{N} 4-\mathrm{H} 4 B \cdots \mathrm{Cl} 2 A^{\mathrm{ii}}$ & 0.91 & 2.64 & $3.4267(15)$ & 146 \\
\hline $\mathrm{N} 4-\mathrm{H} 4 B \cdots \mathrm{O} 1 W^{\mathrm{ii}}$ & 0.91 & 2.39 & $2.9804(17)$ & 123 \\
\hline $\mathrm{N} 5-\mathrm{H} 5 A \cdots \mathrm{Cl} 3 A^{\mathrm{iv}}$ & 0.91 & 2.51 & $3.4245(14)$ & 178 \\
\hline $\mathrm{N} 5-\mathrm{H} 5 A \cdots \mathrm{Cl} 4 B^{\mathrm{iv}}$ & 0.91 & 2.73 & $3.634(19)$ & 173 \\
\hline $\mathrm{N} 5-\mathrm{H} 5 B \cdots \mathrm{Cl} 1 A^{\mathrm{iii}}$ & 0.91 & 2.74 & $3.3664(16)$ & 127 \\
\hline $\mathrm{N} 5-\mathrm{H} 5 B \cdots \mathrm{O} 3 W$ & 0.91 & 2.22 & $2.9724(17)$ & 140 \\
\hline $\mathrm{N} 6-\mathrm{H} 6 A \cdots \mathrm{Cl}^{5}$ & 0.91 & 2.39 & $3.2474(14)$ & 158 \\
\hline $\mathrm{O} 1 W-\mathrm{H} 1 O 1 \cdots \mathrm{Cl} 5$ & $0.85(1)$ & $2.24(1)$ & $3.0878(17)$ & $179(2)$ \\
\hline $\mathrm{O} 1 W-\mathrm{H} 2 O 1 \cdots \mathrm{Cl} 4 A^{\mathrm{ii}}$ & $0.84(1)$ & $2.28(1)$ & $3.1170(13)$ & $174(2)$ \\
\hline $\mathrm{O} 2 W-\mathrm{H} 1 O 2 \cdots \mathrm{Cl} 1 A$ & $0.83(1)$ & $2.28(1)$ & $3.1140(12)$ & $175(2)$ \\
\hline $\mathrm{O} 2 W-\mathrm{H} 1 O 2 \cdots \mathrm{Cl} 2 B$ & $0.83(1)$ & $2.45(1)$ & $3.271(9)$ & $167(2)$ \\
\hline $\mathrm{O} 2 W-\mathrm{H} 2 O 2 \cdots \mathrm{O} 1 W$ & $0.83(1)$ & $1.92(1)$ & $2.7468(19)$ & $177(2)$ \\
\hline $\mathrm{O} 3 W-\mathrm{H} 1 O 3 \cdots \mathrm{Cl}^{\mathrm{iii}}$ & $0.84(1)$ & $2.41(1)$ & $3.2139(13)$ & $159(2)$ \\
\hline $\mathrm{O} 3 W-\mathrm{H} 2 O 3 \cdots \mathrm{Cl} 2 A^{\mathrm{iii}}$ & $0.84(1)$ & $2.38(1)$ & $3.2153(17)$ & $175(2)$ \\
\hline $\mathrm{O} 3 W-\mathrm{H} 2 O 3 \cdots \mathrm{Cl} 3 B^{\mathrm{iii}}$ & $0.84(1)$ & $2.23(2)$ & $3.05(2)$ & $167(2)$ \\
\hline
\end{tabular}

Symmetry codes: (i) $\quad x,-y+\frac{3}{2}, z+\frac{1}{2} ; \quad$ (ii) $\quad-x+2, y+\frac{1}{2},-z+\frac{1}{2}$; $\quad$ (iii) $-x+1, y+\frac{1}{2},-z+\frac{1}{2}$; (iv) $x, y+1, z$.

$\left[\mathrm{Cr}(R R \text {-chxn })_{3}\right]\left(\mathrm{NO}_{3}\right)_{3} \cdot 3 \mathrm{H}_{2} \mathrm{O}$ (Morooka et al., 1992) and $\left[\mathrm{Cr}(\text { rac-chxn })_{3}\right] \mathrm{Cl}_{3} \cdot 2 \mathrm{H}_{2} \mathrm{O}$ (Kalf et al., 2002). The disordered tetrahedral $\left[\mathrm{ZnCl}_{4}\right]^{2-}$ anion, the isolated $\mathrm{Cl}^{-}$anion and the three water molecules remain outside the coordination sphere of $\mathrm{Cr}^{\mathrm{III}}$. The complex $\left[\mathrm{ZnCl}_{4}\right]^{2-}$ anion is distorted due to its involvement in hydrogen-bonding interactions. The $\left[\mathrm{ZnCl}_{4}\right]^{2-}$ and $\mathrm{Cl}^{-}$anions are well separated by van der Waals contacts and consequently there is no basis for describing the $\mathrm{Zn}^{\mathrm{II}}$ species as a distorted $\left[\mathrm{ZnCl}_{5}\right]^{3-}$ anion.

\section{Supramolecular features}

Extensive hydrogen-bonding interactions occur in the crystal structure (Table 1), involving the $\mathrm{N}-\mathrm{H}$ groups of the chxn ligands and the $\mathrm{O}-\mathrm{H}$ groups of the lattice water molecules as donors, and the chloride ions and $\mathrm{Cl}$ atoms of the disordered $\left[\mathrm{ZnCl}_{4}\right]^{2-}$ anions and water $\mathrm{O}$ atoms as acceptors. The supramolecular architecture gives rise to a three-dimensional network structure (Fig. 2).

\section{Database survey}

A search of the Cambridge Structural Database (Version 5.36, May 2015 with last update; Groom et al., 2016) shows that there are three previous reports for $\mathrm{Cr}^{\mathrm{III}}$ complexes containing three chelating chxn ligands, viz. $\left[\mathrm{Cr}(R R \text {-chxn })_{3}\right]\left(\mathrm{NO}_{3}\right)_{3} \cdot 3 \mathrm{H}_{2} \mathrm{O}$ (Morooka et al., 1992), [Cr( rac-chxn) $\left.)_{3}\right] \mathrm{Cl}_{3} \cdot 2 \mathrm{H}_{2} \mathrm{O}$ (Kalf et al., $2002)$, and $\left[\mathrm{Cr}(R R \text {-chxn })_{3}\right]\left[\mathrm{Co}(S S \text {-chxn })_{3}\right] \mathrm{Cl}_{6} \cdot 4 \mathrm{H}_{2} \mathrm{O}$ (Kalf et al., 2002). The structure of any double salt of $\left[\mathrm{Cr}(\operatorname{chxn})_{3}\right]^{3+}$ with an additional $\left[\mathrm{ZnCl}_{4}\right]^{2-}$ anion has not been deposited. 


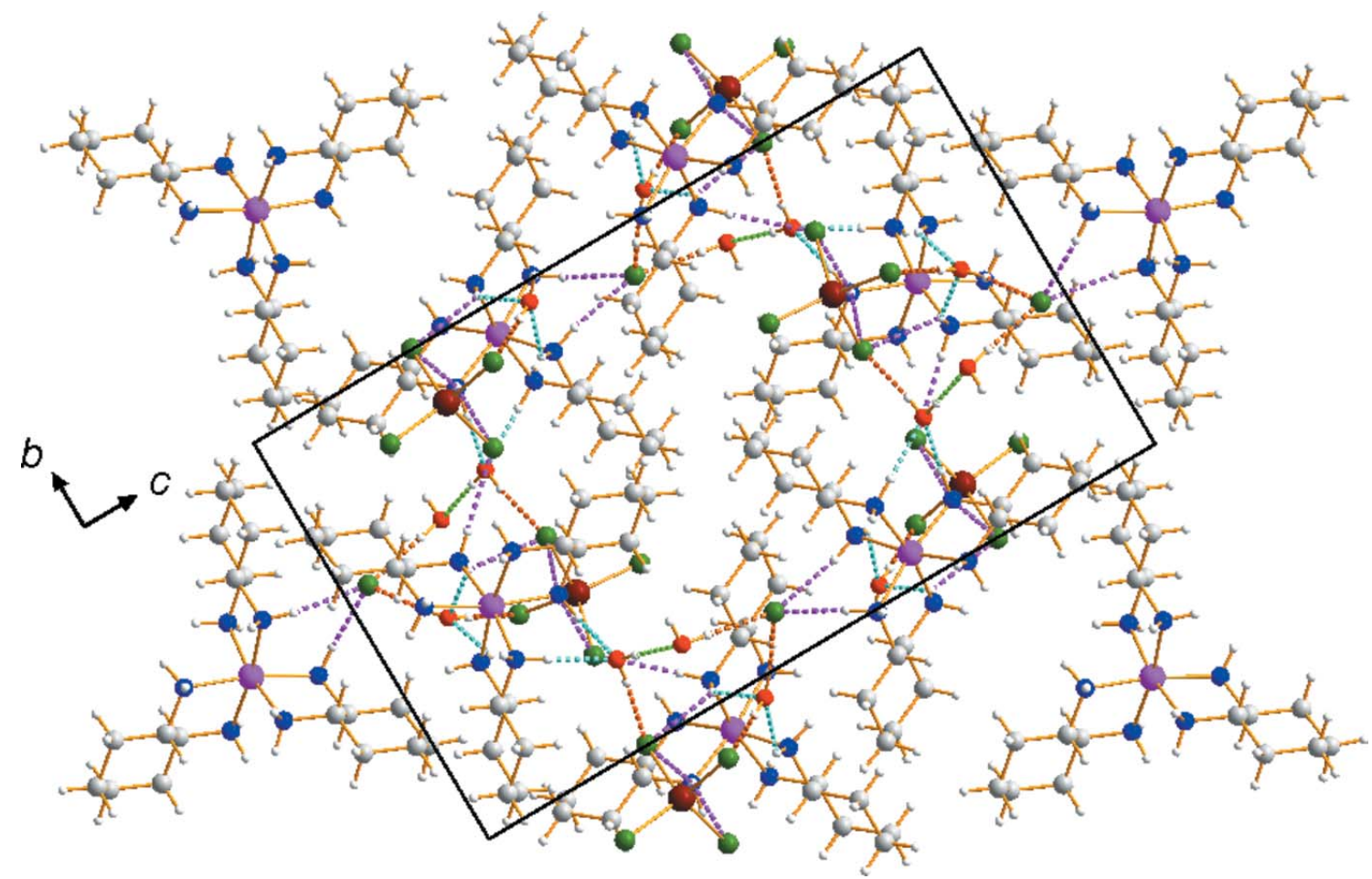

Figure 2

The crystal packing in the title double salt viewed perpendicular to the $b c$ plane. Dashed lines represent hydrogen-bonding interactions: $\mathrm{N}-\mathrm{H} \ldots \mathrm{Cl}$ (pink), $\mathrm{N}-\mathrm{H} \cdots \mathrm{O}$ (cyan), $\mathrm{O}-\mathrm{H} \cdots \mathrm{O}$ (light green) and $\mathrm{O}-\mathrm{H} \cdots \mathrm{Cl}$ (orange). The minor disorder components of the $\left[\mathrm{ZnCl}_{4}\right]^{2-}$ anion have been omitted for clarity.

\section{Synthesis and crystallization}

Commercially available (Aldrich) racemic trans-1,2-cyclohexanediamine was used as provided. All other chemicals with the best analytical grade available were used. The starting material, $\left[\mathrm{Cr}(\text { rac-chxn })_{3}\right] \mathrm{Cl}_{3} \cdot 2 \mathrm{H}_{2} \mathrm{O}$ was prepared according to the literature (Pedersen, 1970). The crude trichloride salt $(0.22 \mathrm{~g})$ was dissolved in $10 \mathrm{~mL}$ of $1 \mathrm{M} \mathrm{HCl}$ at $313 \mathrm{~K}$ and $5 \mathrm{~mL}$ of $1 \mathrm{M} \mathrm{HCl}$ containing $0.5 \mathrm{~g}$ of solid $\mathrm{ZnCl}_{2}$ were added to this solution. The resulting solution was filtered and allowed to stand at room temperature for one week to give block-like yellow crystals of the tetrachloridozincate(II) chloride salt suitable for X-ray structural analysis.

\section{Refinement}

Crystal data, data collection and structure refinement details are summarized in Table 2. All $\mathrm{H}$ atoms were found from difference maps but were placed in geometrically idealized positions and constrained to ride on their parent atoms, with $\mathrm{C}-\mathrm{H}=0.99-1.00 \AA$ and $\mathrm{N}-\mathrm{H}=0.91 \AA$, and with $U_{\text {iso }}(\mathrm{H})$ values of 1.2 or $1.5 U_{\text {eq }}$ of the parent atoms. The hydrogen atoms of water molecules were restrained using DFIX and DANG commands during the least-squares refinement (Sheldrick, 2015b). The $\left[\mathrm{ZnCl}_{4}\right]^{2-}$ anion was refined as positionally disordered over two sets of sites with a refined occupancy ratio constrained to 0.94:0.06 in the last refinement cycles.

Table 2

Experimental details.

Crystal data

Chemical formula

$M_{\mathrm{r}}$

Crystal system, space group

Temperature (K)

$a, b, c(\AA)$

$\beta\left({ }^{\circ}\right)$

$V\left(\AA^{3}\right)$

$Z$

Radiation type

$\mu\left(\mathrm{mm}^{-1}\right)$

Crystal size (mm)

Data collection

Diffractometer

Absorption correction

$T_{\min }, T_{\max }$

No. of measured, independent and observed $[I>2 \sigma(I)]$ reflections

$R_{\text {int }}$

$(\sin \theta / \lambda)_{\max }\left(\AA^{-1}\right)$

Refinement

$R\left[F^{2}>2 \sigma\left(F^{2}\right)\right], w R\left(F^{2}\right), S$

No. of reflections

No. of parameters

No. of restraints

$\mathrm{H}$-atom treatment

$\Delta \rho_{\max }, \Delta \rho_{\min }\left(\mathrm{e} \AA^{-3}\right)$

Computer programs: PAL BL2D-SMDC (Shin et al., 2016), HKL3000sm (Otwinowski \& Minor, 1997), SHELXT2014 (Sheldrick, 2015a), SHELXL2014 (Sheldrick, 2015b), DIAMOND (Putz \& Brandenburg, 2014) and publCIF (Westrip, 2010). 


\section{Acknowledgements}

This work was supported by a grant from 2016 Research Funds of Andong National University. The X-ray crystallography experiment at PLS-II BL2D-SMC beamline was supported in part by MSIP and POSTECH.

\section{References}

Choi, J.-H. (1994). Bull. Korean Chem. Soc. 15, 145-150.

Clegg, W. (1976). Acta Cryst. B32, 2907-2909.

Fabbrizzi, L. \& Poggi, A. (2013). Chem. Soc. Rev. 42, 1681-1699.

Groom, C. R., Bruno, I. J., Lightfoot, M. P. \& Ward, S. C. (2016). Acta Cryst. B72, 171-179.

Kalf, I., Calmuschi, B. \& Englert, U. (2002). CrystEngComm, 4, 548551.

Moon, D. \& Choi, J.-H. (2016). Acta Cryst. E72, 456-459.
Morooka, M., Ohba, S. \& Miyamae, H. (1992). Acta Cryst. B48, 667672.

Otwinowski, Z. \& Minor, W. (1997). Methods in Enzymology, Vol. 276, Macromolecular Crystallography, Part A, edited by C. W. Carter Jr \& R. M. Sweet, pp. 307-326. New York: Academic Press.

Pedersen, E. (1970). Acta Chem. Scand. 24, 3362-3372.

Pons, J., Casabó, J., Palacio, F., Morón, M. C., Solans, X. \& Carlin, R. L. (1988). Inorg. Chim. Acta, 146, 161-165.

Putz, H. \& Brandenburg, K. (2014). DIAMOND. Crystal Impact GbR, Bonn, Germany.

Santos-Figueroa, L. E., Moragues, M. E., Climent, E., Agostini, A., Martínez-Máñez, R. \& Sancenón, F. (2013). Chem. Soc. Rev. 42, 3489-3613.

Sheldrick, G. M. (2015a). Acta Cryst. A71, 3-8.

Sheldrick, G. M. (2015b). Acta Cryst. C71, 3-8.

Shin, J. W., Eom, K. \& Moon, D. (2016). J. Synchrotron Rad. 23, 369373.

Westrip, S. P. (2010). J. Appl. Cryst. 43, 920-925. 


\section{supporting information}

Acta Cryst. (2016). E72, 671-674 [doi:10.1107/S2056989016005788]

Crystal structure of tris(trans-1,2-cyclohexanediamine- $\left.\kappa^{2} N^{\prime} N^{\prime}\right)$ chromium(III) tetrachloridozincate chloride trihydrate from synchrotron data

\section{Dohyun Moon and Jong-Ha Choi}

Computing details

Data collection: PAL BL2D-SMDC (Shin et al., 2016); cell refinement: HKL3000sm (Otwinowski \& Minor, 1997); data reduction: HKL3000sm (Otwinowski \& Minor, 1997); program(s) used to solve structure: SHELXT2014 (Sheldrick, 2015a); program(s) used to refine structure: SHELXL2014 (Sheldrick, 2015b); molecular graphics: DIAMOND (Putz \& Brandenburg, 2014); software used to prepare material for publication: publCIF (Westrip, 2010).

Tris(trans-1,2-cyclohexanediamine- $\kappa^{2} N, N^{\prime}$ ) chromium(III) tetrachloridozincate chloride trihydrate

Crystal data

$\left[\mathrm{Cr}\left(\mathrm{C}_{6} \mathrm{H}_{14} \mathrm{~N}_{2}\right)_{3}\right]\left[\mathrm{ZnCl}_{4}\right] \mathrm{Cl} \cdot 3 \mathrm{H}_{2} \mathrm{O}$

$M_{r}=691.24$

Monoclinic, $P 2_{1} / c$

$a=10.594$ (2) $\AA$

$b=13.075(3) \AA$

$c=22.384(5) \AA$

$\beta=100.87(3)^{\circ}$

$V=3045.0(11) \AA^{3}$

$Z=4$

Data collection

ADSC Q210 CCD area detector diffractometer

Radiation source: PLSII 2D bending magnet

$\omega$ scan

Absorption correction: empirical (using intensity measurements)

(HKL3000sm SCALEPACK; Otwinowski \& Minor, 1997)

$T_{\min }=0.762, T_{\max }=0.945$

Refinement

Refinement on $F^{2}$

Least-squares matrix: full

$R\left[F^{2}>2 \sigma\left(F^{2}\right)\right]=0.027$

$w R\left(F^{2}\right)=0.073$

$S=1.05$

8090 reflections

371 parameters

15 restraints
$F(000)=1444$

$D_{\mathrm{x}}=1.508 \mathrm{Mg} \mathrm{m}^{-3}$

Synchrotron radiation, $\lambda=0.62998 \AA$

Cell parameters from 39315 reflections

$\theta=0.4-33.6^{\circ}$

$\mu=1.15 \mathrm{~mm}^{-1}$

$T=100 \mathrm{~K}$

Block, yellow

$0.25 \times 0.15 \times 0.05 \mathrm{~mm}$

23113 measured reflections

8090 independent reflections

7647 reflections with $I>2 \sigma(I)$

$R_{\text {int }}=0.034$

$\theta_{\max }=26.0^{\circ}, \theta_{\min }=1.7^{\circ}$

$h=-14 \rightarrow 13$

$k=-18 \rightarrow 18$

$l=-31 \rightarrow 23$

Hydrogen site location: mixed

$\mathrm{H}$ atoms treated by a mixture of independent and constrained refinement

$w=1 /\left[\sigma^{2}\left(F_{\mathrm{o}}^{2}\right)+(0.0353 P)^{2}+1.582 P\right]$

where $P=\left(F_{\mathrm{o}}^{2}+2 F_{\mathrm{c}}^{2}\right) / 3$

$(\Delta / \sigma)_{\max }=0.001$

$\Delta \rho_{\max }=1.07 \mathrm{e} \AA^{-3}$

$\Delta \rho_{\min }=-1.14$ e $\AA^{-3}$ 


\section{Special details}

Geometry. All esds (except the esd in the dihedral angle between two 1.s. planes) are estimated using the full covariance matrix. The cell esds are taken into account individually in the estimation of esds in distances, angles and torsion angles; correlations between esds in cell parameters are only used when they are defined by crystal symmetry. An approximate (isotropic) treatment of cell esds is used for estimating esds involving l.s. planes.

Fractional atomic coordinates and isotropic or equivalent isotropic displacement parameters $\left(\AA^{2}\right)$

\begin{tabular}{|c|c|c|c|c|c|}
\hline & $x$ & $y$ & $z$ & $U_{\text {iso }} * / U_{\text {eq }}$ & Occ. $(<1)$ \\
\hline $\mathrm{Cr} 1$ & $0.74469(2)$ & $0.93268(2)$ & $0.34326(2)$ & $0.00945(5)$ & \\
\hline N1 & $0.64213(11)$ & $0.83929(8)$ & $0.39272(5)$ & $0.0148(2)$ & \\
\hline $\mathrm{H} 1 \mathrm{~A}$ & 0.6540 & 0.8610 & 0.4320 & $0.018^{*}$ & \\
\hline H1B & 0.5567 & 0.8427 & 0.3766 & $0.018^{*}$ & \\
\hline $\mathrm{N} 2$ & $0.86651(10)$ & $0.80619(8)$ & $0.35251(5)$ & $0.01418(19)$ & \\
\hline $\mathrm{H} 2 \mathrm{~A}$ & 0.8570 & 0.7719 & 0.3166 & $0.017 *$ & \\
\hline $\mathrm{H} 2 \mathrm{~B}$ & 0.9497 & 0.8270 & 0.3629 & $0.017 *$ & \\
\hline N3 & $0.64039(10)$ & $0.87915(8)$ & $0.26146(5)$ & $0.01276(18)$ & \\
\hline $\mathrm{H} 3 \mathrm{~A}$ & 0.6636 & 0.8136 & 0.2552 & $0.015^{*}$ & \\
\hline H3B & 0.5550 & 0.8799 & 0.2625 & $0.015 *$ & \\
\hline N4 & $0.84308(11)$ & $1.01181(8)$ & $0.28504(5)$ & $0.01452(19)$ & \\
\hline $\mathrm{H} 4 \mathrm{~A}$ & 0.8202 & 1.0790 & 0.2836 & $0.017^{*}$ & \\
\hline H4B & 0.9293 & 1.0076 & 0.2991 & $0.017 *$ & \\
\hline N5 & $0.61717(10)$ & $1.05117(7)$ & $0.35045(5)$ & $0.01305(18)$ & \\
\hline $\mathrm{H} 5 \mathrm{~A}$ & 0.6204 & 1.0989 & 0.3212 & $0.016^{*}$ & \\
\hline $\mathrm{H} 5 \mathrm{~B}$ & 0.5355 & 1.0265 & 0.3453 & $0.016^{*}$ & \\
\hline N6 & $0.85476(11)$ & $1.00563(8)$ & $0.41871(5)$ & $0.0163(2)$ & \\
\hline H6A & 0.8540 & 0.9680 & 0.4529 & $0.020^{*}$ & \\
\hline H6B & 0.9376 & 1.0115 & 0.4135 & $0.020^{*}$ & \\
\hline $\mathrm{C} 1$ & $0.68832(12)$ & $0.73168(8)$ & $0.39046(6)$ & $0.0125(2)$ & \\
\hline H1 & 0.6558 & 0.7035 & 0.3489 & $0.015^{*}$ & \\
\hline $\mathrm{C} 2$ & $0.64271(14)$ & $0.66233(9)$ & $0.43714(6)$ & $0.0179(2)$ & \\
\hline $\mathrm{H} 2 \mathrm{C}$ & 0.5480 & 0.6558 & 0.4273 & $0.021^{*}$ & \\
\hline $\mathrm{H} 2 \mathrm{D}$ & 0.6665 & 0.6929 & 0.4782 & $0.021^{*}$ & \\
\hline $\mathrm{C} 3$ & $0.70450(15)$ & $0.55615(9)$ & $0.43674(6)$ & $0.0208(3)$ & \\
\hline $\mathrm{H} 3 \mathrm{C}$ & 0.6773 & 0.5126 & 0.4682 & $0.025^{*}$ & \\
\hline H3D & 0.6748 & 0.5234 & 0.3967 & $0.025^{*}$ & \\
\hline $\mathrm{C} 4$ & $0.85043(15)$ & $0.56437(10)$ & $0.44909(6)$ & $0.0220(3)$ & \\
\hline $\mathrm{H} 4 \mathrm{C}$ & 0.8885 & 0.4953 & 0.4484 & $0.026^{*}$ & \\
\hline H4D & 0.8806 & 0.5941 & 0.4900 & $0.026^{*}$ & \\
\hline $\mathrm{C} 5$ & $0.89466(13)$ & $0.63175(9)$ & $0.40110(6)$ & $0.0175(2)$ & \\
\hline $\mathrm{H} 5 \mathrm{C}$ & 0.9895 & 0.6380 & 0.4102 & $0.021^{*}$ & \\
\hline $\mathrm{H} 5 \mathrm{D}$ & 0.8694 & 0.5998 & 0.3605 & $0.021 *$ & \\
\hline C6 & $0.83391(12)$ & $0.73752(9)$ & $0.40071(5)$ & $0.0126(2)$ & \\
\hline H6 & 0.8654 & 0.7702 & 0.4412 & $0.015^{*}$ & \\
\hline $\mathrm{C} 7$ & $0.66650(12)$ & $0.94593(8)$ & $0.21109(5)$ & $0.0120(2)$ & \\
\hline $\mathrm{H} 7$ & 0.6215 & 1.0126 & 0.2134 & $0.014 *$ & \\
\hline $\mathrm{C} 8$ & $0.61868(13)$ & $0.89995(10)$ & $0.14856(6)$ & $0.0169(2)$ & \\
\hline H8A & 0.5241 & 0.8921 & 0.1417 & $0.020^{*}$ & \\
\hline
\end{tabular}




\begin{tabular}{|c|c|c|c|c|c|}
\hline H8B & 0.6570 & 0.8313 & 0.1464 & $0.020 *$ & \\
\hline C9 & $0.65520(14)$ & $0.96868(11)$ & $0.09937(6)$ & $0.0221(3)$ & \\
\hline H9A & 0.6104 & 1.0351 & 0.0992 & $0.026^{*}$ & \\
\hline H9B & 0.6273 & 0.9363 & 0.0590 & $0.026^{*}$ & \\
\hline $\mathrm{C} 10$ & $0.80021(14)$ & $0.98656(11)$ & $0.11056(6)$ & $0.0208(3)$ & \\
\hline H10A & 0.8213 & 1.0329 & 0.0789 & $0.025 *$ & \\
\hline H10B & 0.8446 & 0.9207 & 0.1075 & $0.025^{*}$ & \\
\hline C11 & $0.84846(13)$ & $1.03355(9)$ & $0.17347(6)$ & $0.0162(2)$ & \\
\hline H11A & 0.9432 & 1.0405 & 0.1805 & $0.019 *$ & \\
\hline H11B & 0.8110 & 1.1026 & 0.1752 & $0.019 *$ & \\
\hline $\mathrm{C} 12$ & $0.81033(11)$ & $0.96599(9)$ & $0.22289(5)$ & $0.0123(2)$ & \\
\hline H12 & 0.8560 & 0.8990 & 0.2232 & $0.015^{*}$ & \\
\hline C13 & $0.65454(12)$ & $1.09834(9)$ & $0.41220(6)$ & $0.0133(2)$ & \\
\hline H13 & 0.6297 & 1.0503 & 0.4428 & $0.016^{*}$ & \\
\hline $\mathrm{C} 14$ & $0.58832(14)$ & $1.20051(9)$ & $0.41731(7)$ & $0.0199(2)$ & \\
\hline H14A & 0.4940 & 1.1905 & 0.4100 & $0.024 *$ & \\
\hline H14B & 0.6084 & 1.2482 & 0.3861 & $0.024 *$ & \\
\hline $\mathrm{C} 15$ & $0.63376(16)$ & $1.24644(10)$ & $0.48076(7)$ & 0.0253 & \\
\hline $\mathrm{H} 15 \mathrm{~A}$ & 0.5931 & 1.3142 & 0.4829 & $0.030 *$ & \\
\hline H15B & 0.6064 & 1.2016 & 0.5116 & $0.030 *$ & \\
\hline $\mathrm{C} 16$ & 0.77959 (17) & $1.25828(12)$ & $0.49508(8)$ & 0.0303 & \\
\hline H16A & 0.8063 & 1.2837 & 0.5372 & $0.036^{*}$ & \\
\hline H16B & 0.8060 & 1.3094 & 0.4672 & $0.036^{*}$ & \\
\hline $\mathrm{C} 17$ & $0.84742(15)$ & $1.15638(11)$ & $0.48828(7)$ & 0.0255 & \\
\hline H17A & 0.9413 & 1.1678 & 0.4942 & $0.031 *$ & \\
\hline H17B & 0.8309 & 1.1081 & 0.5200 & $0.031 *$ & \\
\hline $\mathrm{C} 18$ & $0.79971(13)$ & $1.10982(9)$ & $0.42553(6)$ & $0.0164(2)$ & \\
\hline H18 & 0.8250 & 1.1561 & 0.3942 & $0.020 *$ & \\
\hline $\mathrm{Zn} 1 \mathrm{~A}$ & $0.76131(2)$ & $0.36294(2)$ & $0.25952(2)$ & $0.01355(6)$ & 0.94 \\
\hline Cl1A & $0.63241(4)$ & 0.50140 & $0.26301(2)$ & $0.01450(9)$ & 0.94 \\
\hline $\mathrm{Cl} 2 \mathrm{~A}$ & $0.86823(7)$ & $0.38639(5)$ & $0.18121(3)$ & $0.02463(12)$ & 0.94 \\
\hline $\mathrm{Cl3A}$ & $0.62548(10)$ & $0.22658(7)$ & $0.23764(4)$ & $0.01813(15)$ & 0.94 \\
\hline Cl4A & $0.89500(4)$ & $0.34751(3)$ & $0.34937(2)$ & $0.02371(8)$ & 0.94 \\
\hline $\mathrm{Zn} 1 \mathrm{~B}$ & $0.7716(6)$ & $0.3345(4)$ & 0.2463 & $0.0287(10)$ & 0.06 \\
\hline Cl1B & $0.9326(9)$ & $0.2641(8)$ & $0.3181(5)$ & $0.056(3)$ & 0.06 \\
\hline $\mathrm{Cl} 2 \mathrm{~B}$ & $0.6709(11)$ & $0.4742(7)$ & $0.2722(6)$ & $0.040(2)$ & 0.06 \\
\hline $\mathrm{Cl3B}$ & $0.8504(18)$ & $0.3625(15)$ & $0.1645(9)$ & $0.062(5)$ & 0.06 \\
\hline C14B & $0.6062(18)$ & $0.2271(16)$ & $0.2239(8)$ & $0.031(4)$ & 0.06 \\
\hline $\mathrm{Cl5}$ & $0.76791(6)$ & $0.60816(3)$ & $0.03343(2)$ & $0.04180(13)$ & \\
\hline $\mathrm{O} 1 \mathrm{~W}$ & $0.94431(12)$ & $0.64959(9)$ & $0.15743(6)$ & $0.0304(3)$ & \\
\hline $\mathrm{H} 1 \mathrm{O} 1$ & 0.8960 (19) & $0.6389(15)$ & $0.1230(6)$ & $0.037 *$ & \\
\hline $\mathrm{H} 2 \mathrm{O} 1$ & $0.982(2)$ & 0.7057 (11) & $0.1558(10)$ & $0.037 *$ & \\
\hline $\mathrm{O} 2 \mathrm{~W}$ & $0.78942(13)$ & $0.69376(8)$ & $0.23958(5)$ & $0.0276(2)$ & \\
\hline $\mathrm{H} 1 \mathrm{O} 2$ & $0.7480(18)$ & 0.6407 (11) & $0.2436(10)$ & $0.033 *$ & \\
\hline $\mathrm{H} 2 \mathrm{O} 2$ & $0.8389(18)$ & $0.6809(15)$ & $0.2159(9)$ & $0.033^{*}$ & \\
\hline $\mathrm{O} 3 \mathrm{~W}$ & $0.40117(10)$ & $0.95853(9)$ & $0.39920(5)$ & $0.0259(2)$ & \\
\hline $\mathrm{H} 1 \mathrm{O} 3$ & 0.3764 (19) & $1.0020(13)$ & $0.4223(8)$ & $0.031 *$ & \\
\hline $\mathrm{H} 2 \mathrm{O} 3$ & $0.3341(14)$ & $0.9374(15)$ & $0.3769(8)$ & $0.031 *$ & \\
\hline
\end{tabular}


Atomic displacement parameters $\left(\AA^{2}\right)$

\begin{tabular}{|c|c|c|c|c|c|c|}
\hline & $U^{11}$ & $U^{22}$ & $U^{33}$ & $U^{12}$ & $U^{13}$ & $U^{23}$ \\
\hline $\mathrm{Cr} 1$ & $0.00941(9)$ & $0.00891(8)$ & $0.01117(9)$ & -0.00020 & $0.00483(7)$ & $0.00071(6)$ \\
\hline N1 & $0.0136(5)$ & $0.0139(4)$ & $0.0196(5)$ & $0.0054(3)$ & $0.0102(4)$ & $0.0066(4)$ \\
\hline N2 & $0.0111(5)$ & 0.0135 & $0.0204(5)$ & $0.0001(3)$ & $0.0094(4)$ & $0.0001(4)$ \\
\hline N3 & $0.0125(5)$ & 0.0125 & $0.0143(5)$ & $-0.0047(3)$ & 0.0050 & $0.0004(3)$ \\
\hline N4 & $0.0151(5)$ & $0.0154(4)$ & $0.0142(5)$ & $-0.0072(4)$ & $0.0057(4)$ & $-0.0016(4)$ \\
\hline N5 & $0.0126(5)$ & $0.0117(4)$ & $0.0145(5)$ & 0.0015 & 0.0019 (4) & $0.0012(3)$ \\
\hline N6 & $0.0112(5)$ & $0.0196(5)$ & $0.0178(5)$ & 0.0035 (4) & 0.0023 (4) & $-0.0034(4)$ \\
\hline $\mathrm{C} 1$ & $0.0119(5)$ & $0.0119(5)$ & $0.0154(5)$ & $0.0019(4)$ & $0.0066(4)$ & $0.0035(4)$ \\
\hline $\mathrm{C} 2$ & $0.0235(7)$ & $0.0145(5)$ & $0.0191(6)$ & 0.0009 (4) & $0.0124(5)$ & $0.0052(4)$ \\
\hline C3 & $0.0355(8)$ & $0.0122(5)$ & $0.0169(6)$ & $0.0012(5)$ & $0.0109(5)$ & $0.0025(4)$ \\
\hline $\mathrm{C} 4$ & $0.0328(8)$ & $0.0146(5)$ & $0.0184(6)$ & $0.0089(5)$ & $0.0042(5)$ & $0.0027(4)$ \\
\hline $\mathrm{C} 5$ & $0.0185(6)$ & $0.0141(5)$ & $0.0205(6)$ & $0.0070(4)$ & $0.0053(5)$ & -0.0009 \\
\hline C6 & $0.0118(5)$ & $0.0117(5)$ & $0.0152(5)$ & $0.0026(4)$ & 0.0045 (4) & $0.0004(4)$ \\
\hline $\mathrm{C} 7$ & $0.0127(5)$ & $0.0122(4)$ & $0.0121(5)$ & -0.0024 & $0.0050(4)$ & 0.0008 (4) \\
\hline $\mathrm{C} 8$ & $0.0162(6)$ & $0.0203(6)$ & $0.0143(5)$ & -0.0045 & $0.0033(4)$ & -0.0025 \\
\hline $\mathrm{C} 9$ & $0.0229(7)$ & $0.0294(7)$ & $0.0139(6)$ & -0.0025 (5) & $0.0038(5)$ & $0.0017(5)$ \\
\hline $\mathrm{C} 10$ & $0.0225(7)$ & $0.0270(6)$ & $0.0149(6)$ & $-0.0026(5)$ & $0.0089(5)$ & $0.0008(5)$ \\
\hline $\mathrm{C} 11$ & $0.0170(6)$ & $0.0176(5)$ & $0.0162(5)$ & -0.0049 & $0.0088(4)$ & $0.0019(4)$ \\
\hline $\mathrm{C} 12$ & $0.0127(5)$ & $0.0126(5)$ & $0.0130(5)$ & $-0.0030(4)$ & $0.0058(4)$ & $-0.0004(4)$ \\
\hline $\mathrm{C} 13$ & $0.0140(5)$ & $0.0108(5)$ & $0.0156(5)$ & 0.0015 & $0.0041(4)$ & $-0.0001(4)$ \\
\hline $\mathrm{C} 14$ & $0.0227(6)$ & $0.0123(5)$ & $0.0265(7)$ & $0.0055(4)$ & $0.0096(5)$ & $0.0000(4)$ \\
\hline $\mathrm{C} 15$ & $0.0329(8)$ & $0.0169(6)$ & $0.0294(7)$ & $0.0023(5)$ & $0.0145(6)$ & $-0.0063(5)$ \\
\hline $\mathrm{C} 16$ & $0.0357(9)$ & $0.0223(6)$ & $0.0345(8)$ & $-0.0063(6)$ & $0.0104(7)$ & $-0.0146(6)$ \\
\hline $\mathrm{C} 17$ & $0.0231(7)$ & $0.0269(7)$ & $0.0256(7)$ & $-0.0030(5)$ & $0.0020(6)$ & $-0.0132(5)$ \\
\hline C18 & $0.0149(6)$ & $0.0145(5)$ & $0.0202(6)$ & -0.0010 & $0.0047(4)$ & $-0.0048(4)$ \\
\hline $\mathrm{Zn} 1 \mathrm{~A}$ & $0.00992(9)$ & $0.01085(11)$ & $0.02095(13)$ & $0.00062(7)$ & $0.00562(8)$ & $0.00178(7)$ \\
\hline Cl1A & $0.01152(18)$ & $0.01180(19)$ & $0.02058(16)$ & 0.00198 (12) & $0.00407(13)$ & $-0.00062(13)$ \\
\hline $\mathrm{Cl} 2 \mathrm{~A}$ & $0.0129(2)$ & $0.0329(3)$ & $0.0317(3)$ & $0.00436(17)$ & $0.0133(2)$ & $0.0052(2)$ \\
\hline $\mathrm{Cl3A}$ & $0.0171(4)$ & 0.01019 (19) & $0.0277(4)$ & $-0.0013(2)$ & 0.0058 & $-0.0002(2)$ \\
\hline Cl4A & $0.02115(17)$ & $0.01976(15)$ & $0.02746(18)$ & $0.00188(11)$ & $-0.00247(13)$ & $0.00251(12)$ \\
\hline $\mathrm{Zn} 1 \mathrm{~B}$ & $0.029(2)$ & $0.027(2)$ & $0.034(3)$ & $-0.0094(19)$ & $0.0149(16)$ & $-0.0010(17)$ \\
\hline Cl1B & $0.037(4)$ & $0.069(6)$ & $0.059(6)$ & -0.013 & $-0.002(4)$ & $0.028(5)$ \\
\hline $\mathrm{Cl} 2 \mathrm{~B}$ & $0.037(5)$ & $0.019(4)$ & $0.071(7)$ & $-0.017(3)$ & $0.030(5)$ & -0.020 \\
\hline $\mathrm{Cl3B}$ & $0.041(9)$ & 0.085 (12) & $0.066(11)$ & $0.026(7)$ & $0.028(8)$ & $-0.001(7)$ \\
\hline C14B & $0.010(4)$ & $0.034(5)$ & $0.045(8)$ & $0.010(3)$ & $-0.008(4)$ & $-0.004(5)$ \\
\hline $\mathrm{Cl} 5$ & 0.0815 & $0.03036(19)$ & $0.01572(16)$ & $-0.0234(2)$ & 0.01466 (19) & $-0.00282(13)$ \\
\hline $\mathrm{O} 1 \mathrm{~W}$ & $0.0211(5)$ & $0.0298(5)$ & $0.0381(7)$ & $-0.0013(4)$ & $0.0000(5)$ & $0.0111(5)$ \\
\hline $\mathrm{O} 2 \mathrm{~W}$ & $0.0423(7)$ & $0.0146(4)$ & $0.0300(6)$ & $-0.0058(4)$ & $0.0168(5)$ & $-0.0031(4)$ \\
\hline $\mathrm{O} 3 \mathrm{~W}$ & $0.0156(5)$ & $0.0347(6)$ & $0.0285(5)$ & $0.0001(4)$ & $0.0071(4)$ & $-0.0024(4)$ \\
\hline
\end{tabular}

Geometric parameters $\left(\AA,{ }^{\circ}\right)$

\begin{tabular}{llll}
\hline Cr1-N3 & $2.0737(12)$ & C8-H8A & 0.9900 \\
Cr1-N5 & $2.0817(10)$ & C8-H8B & 0.9900 \\
Cr1-N2 & $2.0839(11)$ & C9-C10 & $1.527(2)$
\end{tabular}




\begin{tabular}{|c|c|c|c|}
\hline $\mathrm{Cr} 1-\mathrm{N} 1$ & $2.0859(11)$ & C9-H9A & 0.9900 \\
\hline $\mathrm{Cr} 1-\mathrm{N} 4$ & $2.0899(11)$ & C9-H9B & 0.9900 \\
\hline $\mathrm{Cr} 1-\mathrm{N} 6$ & $2.0928(12)$ & $\mathrm{C} 10-\mathrm{C} 11$ & $1.5330(19)$ \\
\hline $\mathrm{N} 1-\mathrm{C} 1$ & $1.4937(15)$ & $\mathrm{C} 10-\mathrm{H} 10 \mathrm{~A}$ & 0.9900 \\
\hline $\mathrm{N} 1-\mathrm{H} 1 \mathrm{~A}$ & 0.9100 & $\mathrm{C} 10-\mathrm{H} 10 \mathrm{~B}$ & 0.9900 \\
\hline N1-H1B & 0.9100 & $\mathrm{C} 11-\mathrm{C} 12$ & $1.5283(16)$ \\
\hline $\mathrm{N} 2-\mathrm{C} 6$ & $1.4932(15)$ & $\mathrm{C} 11-\mathrm{H} 11 \mathrm{~A}$ & 0.9900 \\
\hline $\mathrm{N} 2-\mathrm{H} 2 \mathrm{~A}$ & 0.9100 & C11-H11B & 0.9900 \\
\hline $\mathrm{N} 2-\mathrm{H} 2 \mathrm{~B}$ & 0.9100 & $\mathrm{C} 12-\mathrm{H} 12$ & 1.0000 \\
\hline $\mathrm{N} 3-\mathrm{C} 7$ & $1.4927(15)$ & $\mathrm{C} 13-\mathrm{C} 18$ & $1.5178(18)$ \\
\hline $\mathrm{N} 3-\mathrm{H} 3 \mathrm{~A}$ & 0.9100 & $\mathrm{C} 13-\mathrm{C} 14$ & $1.5232(16)$ \\
\hline N3-H3B & 0.9100 & $\mathrm{C} 13-\mathrm{H} 13$ & 1.0000 \\
\hline $\mathrm{N} 4-\mathrm{C} 12$ & $1.4942(15)$ & $\mathrm{C} 14-\mathrm{C} 15$ & $1.533(2)$ \\
\hline $\mathrm{N} 4-\mathrm{H} 4 \mathrm{~A}$ & 0.9100 & $\mathrm{C} 14-\mathrm{H} 14 \mathrm{~A}$ & 0.9900 \\
\hline $\mathrm{N} 4-\mathrm{H} 4 \mathrm{~B}$ & 0.9100 & C14-H14B & 0.9900 \\
\hline $\mathrm{N} 5-\mathrm{C} 13$ & $1.4971(16)$ & $\mathrm{C} 15-\mathrm{C} 16$ & $1.525(2)$ \\
\hline N5-H5A & 0.9100 & $\mathrm{C} 15-\mathrm{H} 15 \mathrm{~A}$ & 0.9900 \\
\hline N5-H5B & 0.9100 & C15-H15B & 0.9900 \\
\hline $\mathrm{N} 6-\mathrm{C} 18$ & $1.5009(16)$ & $\mathrm{C} 16-\mathrm{C} 17$ & $1.535(2)$ \\
\hline $\mathrm{N} 6-\mathrm{H} 6 \mathrm{~A}$ & 0.9100 & $\mathrm{C} 16-\mathrm{H} 16 \mathrm{~A}$ & 0.9900 \\
\hline N6-H6B & 0.9100 & C16-H16B & 0.9900 \\
\hline $\mathrm{C} 1-\mathrm{C} 6$ & $1.5179(17)$ & $\mathrm{C} 17-\mathrm{C} 18$ & $1.5270(19)$ \\
\hline $\mathrm{C} 1-\mathrm{C} 2$ & $1.5289(16)$ & C17-H17A & 0.9900 \\
\hline $\mathrm{C} 1-\mathrm{H} 1$ & 1.0000 & C17-H17B & 0.9900 \\
\hline $\mathrm{C} 2-\mathrm{C} 3$ & $1.5356(18)$ & $\mathrm{C} 18-\mathrm{H} 18$ & 1.0000 \\
\hline $\mathrm{C} 2-\mathrm{H} 2 \mathrm{C}$ & 0.9900 & $\mathrm{Zn} 1 \mathrm{~A}-\mathrm{Cl} 4 \mathrm{~A}$ & $2.2400(9)$ \\
\hline $\mathrm{C} 2-\mathrm{H} 2 \mathrm{D}$ & 0.9900 & $\mathrm{Zn} 1 \mathrm{~A}-\mathrm{Cl1A}$ & $2.2779(6)$ \\
\hline $\mathrm{C} 3-\mathrm{C} 4$ & $1.522(2)$ & $\mathrm{Zn} 1 \mathrm{~A}-\mathrm{Cl} 2 \mathrm{~A}$ & $2.2799(9)$ \\
\hline $\mathrm{C} 3-\mathrm{H} 3 \mathrm{C}$ & 0.9900 & $\mathrm{Zn} 1 \mathrm{~A}-\mathrm{Cl} 3 \mathrm{~A}$ & $2.2856(10)$ \\
\hline $\mathrm{C} 3-\mathrm{H} 3 \mathrm{D}$ & 0.9900 & $\mathrm{Zn} 1 \mathrm{~B}-\mathrm{Cl3B}$ & $2.18(2)$ \\
\hline $\mathrm{C} 4-\mathrm{C} 5$ & $1.529(2)$ & $\mathrm{Zn} 1 \mathrm{~B}-\mathrm{Cl} 14 \mathrm{~B}$ & $2.23(2)$ \\
\hline $\mathrm{C} 4-\mathrm{H} 4 \mathrm{C}$ & 0.9900 & $\mathrm{Zn} 1 \mathrm{~B}-\mathrm{Cl} 2 \mathrm{~B}$ & $2.246(11)$ \\
\hline $\mathrm{C} 4-\mathrm{H} 4 \mathrm{D}$ & 0.9900 & $\mathrm{Zn} 1 \mathrm{~B}-\mathrm{Cl1B}$ & $2.304(11)$ \\
\hline $\mathrm{C} 5-\mathrm{C} 6$ & $1.5247(16)$ & $\mathrm{Cl1B}-\mathrm{O} 1 \mathrm{~W}^{\mathrm{i}}$ & $1.993(11)$ \\
\hline $\mathrm{C} 5-\mathrm{H} 5 \mathrm{C}$ & 0.9900 & $\mathrm{O} 1 \mathrm{~W}-\mathrm{C} 11 \mathrm{~B}^{\mathrm{ii}}$ & $1.993(11)$ \\
\hline C5-H5D & 0.9900 & $\mathrm{O} 1 \mathrm{~W}-\mathrm{H} 1 \mathrm{O} 1$ & $0.853(9)$ \\
\hline $\mathrm{C} 6-\mathrm{H} 6$ & 1.0000 & $\mathrm{O} 1 \mathrm{~W}-\mathrm{H} 2 \mathrm{O} 1$ & $0.841(9)$ \\
\hline $\mathrm{C} 7-\mathrm{C} 12$ & 1.5194 (17) & $\mathrm{O} 2 \mathrm{~W}-\mathrm{H} 1 \mathrm{O} 2$ & $0.834(9)$ \\
\hline $\mathrm{C} 7-\mathrm{C} 8$ & $1.5197(17)$ & $\mathrm{O} 2 \mathrm{~W}-\mathrm{H} 2 \mathrm{O} 2$ & $0.829(9)$ \\
\hline $\mathrm{C} 7-\mathrm{H} 7$ & 1.0000 & $\mathrm{O} 3 \mathrm{~W}-\mathrm{H} 1 \mathrm{O} 3$ & $0.843(9)$ \\
\hline $\mathrm{C} 8-\mathrm{C} 9$ & $1.5267(19)$ & $\mathrm{O} 3 \mathrm{~W}-\mathrm{H} 2 \mathrm{O} 3$ & $0.835(9)$ \\
\hline $\mathrm{N} 3-\mathrm{Cr} 1-\mathrm{N} 5$ & $94.24(5)$ & $\mathrm{C} 12-\mathrm{C} 7-\mathrm{C} 8$ & $112.19(10)$ \\
\hline $\mathrm{N} 3-\mathrm{Cr} 1-\mathrm{N} 2$ & $92.11(5)$ & $\mathrm{N} 3-\mathrm{C} 7-\mathrm{H} 7$ & 108.2 \\
\hline $\mathrm{N} 5-\mathrm{Cr} 1-\mathrm{N} 2$ & $169.03(4)$ & $\mathrm{C} 12-\mathrm{C} 7-\mathrm{H} 7$ & 108.2 \\
\hline $\mathrm{N} 3-\mathrm{Cr} 1-\mathrm{N} 1$ & $91.55(5)$ & $\mathrm{C} 8-\mathrm{C} 7-\mathrm{H} 7$ & 108.2 \\
\hline $\mathrm{N} 5-\mathrm{Cr} 1-\mathrm{N} 1$ & 89.09 (4) & $\mathrm{C} 7-\mathrm{C} 8-\mathrm{C} 9$ & $110.21(10)$ \\
\hline $\mathrm{N} 2-\mathrm{Cr} 1-\mathrm{N} 1$ & $81.81(4)$ & $\mathrm{C} 7-\mathrm{C} 8-\mathrm{H} 8 \mathrm{~A}$ & 109.6 \\
\hline
\end{tabular}




\begin{tabular}{|c|c|c|c|}
\hline $\mathrm{N} 3-\mathrm{Cr} 1-\mathrm{N} 4$ & $82.06(4)$ & $\mathrm{C} 9-\mathrm{C} 8-\mathrm{H} 8 \mathrm{~A}$ & 109.6 \\
\hline $\mathrm{N} 5-\mathrm{Cr} 1-\mathrm{N} 4$ & $94.99(5)$ & $\mathrm{C} 7-\mathrm{C} 8-\mathrm{H} 8 \mathrm{~B}$ & 109.6 \\
\hline $\mathrm{N} 2-\mathrm{Cr} 1-\mathrm{N} 4$ & $94.74(5)$ & $\mathrm{C} 9-\mathrm{C} 8-\mathrm{H} 8 \mathrm{~B}$ & 109.6 \\
\hline $\mathrm{N} 1-\mathrm{Cr} 1-\mathrm{N} 4$ & $172.64(4)$ & $\mathrm{H} 8 \mathrm{~A}-\mathrm{C} 8-\mathrm{H} 8 \mathrm{~B}$ & 108.1 \\
\hline $\mathrm{N} 3-\mathrm{Cr} 1-\mathrm{N} 6$ & $171.68(4)$ & $\mathrm{C} 8-\mathrm{C} 9-\mathrm{C} 10$ & $110.83(12)$ \\
\hline $\mathrm{N} 5-\mathrm{Cr} 1-\mathrm{N} 6$ & $82.49(5)$ & $\mathrm{C} 8-\mathrm{C} 9-\mathrm{H} 9 \mathrm{~A}$ & 109.5 \\
\hline $\mathrm{N} 2-\mathrm{Cr} 1-\mathrm{N} 6$ & $92.35(5)$ & $\mathrm{C} 10-\mathrm{C} 9-\mathrm{H} 9 \mathrm{~A}$ & 109.5 \\
\hline $\mathrm{N} 1-\mathrm{Cr} 1-\mathrm{N} 6$ & $96.04(5)$ & $\mathrm{C} 8-\mathrm{C} 9-\mathrm{H} 9 \mathrm{~B}$ & 109.5 \\
\hline $\mathrm{N} 4-\mathrm{Cr} 1-\mathrm{N} 6$ & $90.58(5)$ & $\mathrm{C} 10-\mathrm{C} 9-\mathrm{H} 9 \mathrm{~B}$ & 109.5 \\
\hline $\mathrm{C} 1-\mathrm{N} 1-\mathrm{Cr} 1$ & $109.07(7)$ & $\mathrm{H} 9 \mathrm{~A}-\mathrm{C} 9-\mathrm{H} 9 \mathrm{~B}$ & 108.1 \\
\hline $\mathrm{C} 1-\mathrm{N} 1-\mathrm{H} 1 \mathrm{~A}$ & 109.9 & $\mathrm{C} 9-\mathrm{C} 10-\mathrm{C} 11$ & $111.28(11)$ \\
\hline $\mathrm{Cr} 1-\mathrm{N} 1-\mathrm{H} 1 \mathrm{~A}$ & 109.9 & $\mathrm{C} 9-\mathrm{C} 10-\mathrm{H} 10 \mathrm{~A}$ & 109.4 \\
\hline $\mathrm{C} 1-\mathrm{N} 1-\mathrm{H} 1 \mathrm{~B}$ & 109.9 & $\mathrm{C} 11-\mathrm{C} 10-\mathrm{H} 10 \mathrm{~A}$ & 109.4 \\
\hline $\mathrm{Cr} 1-\mathrm{N} 1-\mathrm{H} 1 \mathrm{~B}$ & 109.9 & C9- $\mathrm{C} 10-\mathrm{H} 10 \mathrm{~B}$ & 109.4 \\
\hline $\mathrm{H} 1 \mathrm{~A}-\mathrm{N} 1-\mathrm{H} 1 \mathrm{~B}$ & 108.3 & $\mathrm{C} 11-\mathrm{C} 10-\mathrm{H} 10 \mathrm{~B}$ & 109.4 \\
\hline $\mathrm{C} 6-\mathrm{N} 2-\mathrm{Cr} 1$ & $109.01(7)$ & $\mathrm{H} 10 \mathrm{~A}-\mathrm{C} 10-\mathrm{H} 10 \mathrm{~B}$ & 108.0 \\
\hline $\mathrm{C} 6-\mathrm{N} 2-\mathrm{H} 2 \mathrm{~A}$ & 109.9 & $\mathrm{C} 12-\mathrm{C} 11-\mathrm{C} 10$ & $110.21(10)$ \\
\hline $\mathrm{Cr} 1-\mathrm{N} 2-\mathrm{H} 2 \mathrm{~A}$ & 109.9 & $\mathrm{C} 12-\mathrm{C} 11-\mathrm{H} 11 \mathrm{~A}$ & 109.6 \\
\hline $\mathrm{C} 6-\mathrm{N} 2-\mathrm{H} 2 \mathrm{~B}$ & 109.9 & $\mathrm{C} 10-\mathrm{C} 11-\mathrm{H} 11 \mathrm{~A}$ & 109.6 \\
\hline $\mathrm{Cr} 1-\mathrm{N} 2-\mathrm{H} 2 \mathrm{~B}$ & 109.9 & $\mathrm{C} 12-\mathrm{C} 11-\mathrm{H} 11 \mathrm{~B}$ & 109.6 \\
\hline $\mathrm{H} 2 \mathrm{~A}-\mathrm{N} 2-\mathrm{H} 2 \mathrm{~B}$ & 108.3 & $\mathrm{C} 10-\mathrm{C} 11-\mathrm{H} 11 \mathrm{~B}$ & 109.6 \\
\hline $\mathrm{C} 7-\mathrm{N} 3-\mathrm{Cr} 1$ & $109.01(7)$ & $\mathrm{H} 11 \mathrm{~A}-\mathrm{C} 11-\mathrm{H} 11 \mathrm{~B}$ & 108.1 \\
\hline $\mathrm{C} 7-\mathrm{N} 3-\mathrm{H} 3 \mathrm{~A}$ & 109.9 & $\mathrm{~N} 4-\mathrm{C} 12-\mathrm{C} 7$ & $106.28(10)$ \\
\hline $\mathrm{Cr} 1-\mathrm{N} 3-\mathrm{H} 3 \mathrm{~A}$ & 109.9 & $\mathrm{~N} 4-\mathrm{C} 12-\mathrm{C} 11$ & $113.29(9)$ \\
\hline $\mathrm{C} 7-\mathrm{N} 3-\mathrm{H} 3 \mathrm{~B}$ & 109.9 & $\mathrm{C} 7-\mathrm{C} 12-\mathrm{C} 11$ & $111.54(10)$ \\
\hline $\mathrm{Cr} 1-\mathrm{N} 3-\mathrm{H} 3 \mathrm{~B}$ & 109.9 & $\mathrm{~N} 4-\mathrm{C} 12-\mathrm{H} 12$ & 108.5 \\
\hline $\mathrm{H} 3 \mathrm{~A}-\mathrm{N} 3-\mathrm{H} 3 \mathrm{~B}$ & 108.3 & $\mathrm{C} 7-\mathrm{C} 12-\mathrm{H} 12$ & 108.5 \\
\hline $\mathrm{C} 12-\mathrm{N} 4-\mathrm{Cr} 1$ & $109.05(7)$ & $\mathrm{C} 11-\mathrm{C} 12-\mathrm{H} 12$ & 108.5 \\
\hline $\mathrm{C} 12-\mathrm{N} 4-\mathrm{H} 4 \mathrm{~A}$ & 109.9 & $\mathrm{~N} 5-\mathrm{C} 13-\mathrm{C} 18$ & $107.70(10)$ \\
\hline $\mathrm{Cr} 1-\mathrm{N} 4-\mathrm{H} 4 \mathrm{~A}$ & 109.9 & $\mathrm{~N} 5-\mathrm{C} 13-\mathrm{C} 14$ & $112.66(10)$ \\
\hline $\mathrm{C} 12-\mathrm{N} 4-\mathrm{H} 4 \mathrm{~B}$ & 109.9 & $\mathrm{C} 18-\mathrm{C} 13-\mathrm{C} 14$ & $111.25(10)$ \\
\hline $\mathrm{Cr} 1-\mathrm{N} 4-\mathrm{H} 4 \mathrm{~B}$ & 109.9 & $\mathrm{~N} 5-\mathrm{C} 13-\mathrm{H} 13$ & 108.4 \\
\hline $\mathrm{H} 4 \mathrm{~A}-\mathrm{N} 4-\mathrm{H} 4 \mathrm{~B}$ & 108.3 & $\mathrm{C} 18-\mathrm{C} 13-\mathrm{H} 13$ & 108.4 \\
\hline $\mathrm{C} 13-\mathrm{N} 5-\mathrm{Cr} 1$ & $108.39(7)$ & $\mathrm{C} 14-\mathrm{C} 13-\mathrm{H} 13$ & 108.4 \\
\hline $\mathrm{C} 13-\mathrm{N} 5-\mathrm{H} 5 \mathrm{~A}$ & 110.0 & $\mathrm{C} 13-\mathrm{C} 14-\mathrm{C} 15$ & $110.15(11)$ \\
\hline $\mathrm{Cr} 1-\mathrm{N} 5-\mathrm{H} 5 \mathrm{~A}$ & 110.0 & $\mathrm{C} 13-\mathrm{C} 14-\mathrm{H} 14 \mathrm{~A}$ & 109.6 \\
\hline $\mathrm{C} 13-\mathrm{N} 5-\mathrm{H} 5 \mathrm{~B}$ & 110.0 & $\mathrm{C} 15-\mathrm{C} 14-\mathrm{H} 14 \mathrm{~A}$ & 109.6 \\
\hline $\mathrm{Cr} 1-\mathrm{N} 5-\mathrm{H} 5 \mathrm{~B}$ & 110.0 & $\mathrm{C} 13-\mathrm{C} 14-\mathrm{H} 14 \mathrm{~B}$ & 109.6 \\
\hline $\mathrm{H} 5 \mathrm{~A}-\mathrm{N} 5-\mathrm{H} 5 \mathrm{~B}$ & 108.4 & $\mathrm{C} 15-\mathrm{C} 14-\mathrm{H} 14 \mathrm{~B}$ & 109.6 \\
\hline $\mathrm{C} 18-\mathrm{N} 6-\mathrm{Cr} 1$ & $109.12(8)$ & $\mathrm{H} 14 \mathrm{~A}-\mathrm{C} 14-\mathrm{H} 14 \mathrm{~B}$ & 108.1 \\
\hline $\mathrm{C} 18-\mathrm{N} 6-\mathrm{H} 6 \mathrm{~A}$ & 109.9 & $\mathrm{C} 16-\mathrm{C} 15-\mathrm{C} 14$ & $111.29(12)$ \\
\hline $\mathrm{Cr} 1-\mathrm{N} 6-\mathrm{H} 6 \mathrm{~A}$ & 109.9 & $\mathrm{C} 16-\mathrm{C} 15-\mathrm{H} 15 \mathrm{~A}$ & 109.4 \\
\hline $\mathrm{C} 18-\mathrm{N} 6-\mathrm{H} 6 \mathrm{~B}$ & 109.9 & $\mathrm{C} 14-\mathrm{C} 15-\mathrm{H} 15 \mathrm{~A}$ & 109.4 \\
\hline $\mathrm{Cr} 1-\mathrm{N} 6-\mathrm{H} 6 \mathrm{~B}$ & 109.9 & $\mathrm{C} 16-\mathrm{C} 15-\mathrm{H} 15 \mathrm{~B}$ & 109.4 \\
\hline $\mathrm{H} 6 \mathrm{~A}-\mathrm{N} 6-\mathrm{H} 6 \mathrm{~B}$ & 108.3 & $\mathrm{C} 14-\mathrm{C} 15-\mathrm{H} 15 \mathrm{~B}$ & 109.4 \\
\hline $\mathrm{N} 1-\mathrm{C} 1-\mathrm{C} 6$ & $106.11(9)$ & $\mathrm{H} 15 \mathrm{~A}-\mathrm{C} 15-\mathrm{H} 15 \mathrm{~B}$ & 108.0 \\
\hline $\mathrm{N} 1-\mathrm{C} 1-\mathrm{C} 2$ & $112.82(10)$ & $\mathrm{C} 15-\mathrm{C} 16-\mathrm{C} 17$ & $111.45(12)$ \\
\hline $\mathrm{C} 6-\mathrm{C} 1-\mathrm{C} 2$ & $111.69(10)$ & $\mathrm{C} 15-\mathrm{C} 16-\mathrm{H} 16 \mathrm{~A}$ & 109.3 \\
\hline
\end{tabular}




\begin{tabular}{|c|c|c|c|}
\hline $\mathrm{N} 1-\mathrm{C} 1-\mathrm{H} 1$ & 108.7 & $\mathrm{C} 17-\mathrm{C} 16-\mathrm{H} 16 \mathrm{~A}$ & 109.3 \\
\hline $\mathrm{C} 6-\mathrm{C} 1-\mathrm{H} 1$ & 108.7 & $\mathrm{C} 15-\mathrm{C} 16-\mathrm{H} 16 \mathrm{~B}$ & 109.3 \\
\hline $\mathrm{C} 2-\mathrm{C} 1-\mathrm{H} 1$ & 108.7 & $\mathrm{C} 17-\mathrm{C} 16-\mathrm{H} 16 \mathrm{~B}$ & 109.3 \\
\hline $\mathrm{C} 1-\mathrm{C} 2-\mathrm{C} 3$ & $110.02(10)$ & $\mathrm{H} 16 \mathrm{~A}-\mathrm{C} 16-\mathrm{H} 16 \mathrm{~B}$ & 108.0 \\
\hline $\mathrm{C} 1-\mathrm{C} 2-\mathrm{H} 2 \mathrm{C}$ & 109.7 & $\mathrm{C} 18-\mathrm{C} 17-\mathrm{C} 16$ & $110.94(13)$ \\
\hline $\mathrm{C} 3-\mathrm{C} 2-\mathrm{H} 2 \mathrm{C}$ & 109.7 & $\mathrm{C} 18-\mathrm{C} 17-\mathrm{H} 17 \mathrm{~A}$ & 109.5 \\
\hline $\mathrm{C} 1-\mathrm{C} 2-\mathrm{H} 2 \mathrm{D}$ & 109.7 & $\mathrm{C} 16-\mathrm{C} 17-\mathrm{H} 17 \mathrm{~A}$ & 109.5 \\
\hline $\mathrm{C} 3-\mathrm{C} 2-\mathrm{H} 2 \mathrm{D}$ & 109.7 & $\mathrm{C} 18-\mathrm{C} 17-\mathrm{H} 17 \mathrm{~B}$ & 109.5 \\
\hline $\mathrm{H} 2 \mathrm{C}-\mathrm{C} 2-\mathrm{H} 2 \mathrm{D}$ & 108.2 & $\mathrm{C} 16-\mathrm{C} 17-\mathrm{H} 17 \mathrm{~B}$ & 109.5 \\
\hline $\mathrm{C} 4-\mathrm{C} 3-\mathrm{C} 2$ & $110.76(11)$ & $\mathrm{H} 17 \mathrm{~A}-\mathrm{C} 17-\mathrm{H} 17 \mathrm{~B}$ & 108.0 \\
\hline $\mathrm{C} 4-\mathrm{C} 3-\mathrm{H} 3 \mathrm{C}$ & 109.5 & $\mathrm{~N} 6-\mathrm{C} 18-\mathrm{C} 13$ & $106.80(10)$ \\
\hline $\mathrm{C} 2-\mathrm{C} 3-\mathrm{H} 3 \mathrm{C}$ & 109.5 & $\mathrm{~N} 6-\mathrm{C} 18-\mathrm{C} 17$ & $112.82(11)$ \\
\hline $\mathrm{C} 4-\mathrm{C} 3-\mathrm{H} 3 \mathrm{D}$ & 109.5 & $\mathrm{C} 13-\mathrm{C} 18-\mathrm{C} 17$ & $111.57(11)$ \\
\hline $\mathrm{C} 2-\mathrm{C} 3-\mathrm{H} 3 \mathrm{D}$ & 109.5 & $\mathrm{~N} 6-\mathrm{C} 18-\mathrm{H} 18$ & 108.5 \\
\hline $\mathrm{H} 3 \mathrm{C}-\mathrm{C} 3-\mathrm{H} 3 \mathrm{D}$ & 108.1 & $\mathrm{C} 13-\mathrm{C} 18-\mathrm{H} 18$ & 108.5 \\
\hline $\mathrm{C} 3-\mathrm{C} 4-\mathrm{C} 5$ & $110.38(11)$ & $\mathrm{C} 17-\mathrm{C} 18-\mathrm{H} 18$ & 108.5 \\
\hline $\mathrm{C} 3-\mathrm{C} 4-\mathrm{H} 4 \mathrm{C}$ & 109.6 & $\mathrm{Cl} 14 \mathrm{~A}-\mathrm{Zn} 1 \mathrm{~A}-\mathrm{Cl1} \mathrm{A}$ & $108.81(2)$ \\
\hline $\mathrm{C} 5-\mathrm{C} 4-\mathrm{H} 4 \mathrm{C}$ & 109.6 & $\mathrm{Cl} 4 \mathrm{~A}-\mathrm{Zn} 1 \mathrm{~A}-\mathrm{Cl} 2 \mathrm{~A}$ & $112.38(3)$ \\
\hline $\mathrm{C} 3-\mathrm{C} 4-\mathrm{H} 4 \mathrm{D}$ & 109.6 & $\mathrm{C} 11 \mathrm{~A}-\mathrm{Zn} 1 \mathrm{~A}-\mathrm{Cl} 2 \mathrm{~A}$ & $107.91(3)$ \\
\hline $\mathrm{C} 5-\mathrm{C} 4-\mathrm{H} 4 \mathrm{D}$ & 109.6 & $\mathrm{Cl} 14 \mathrm{~A}-\mathrm{Zn} 1 \mathrm{~A}-\mathrm{Cl} 3 \mathrm{~A}$ & $112.84(3)$ \\
\hline $\mathrm{H} 4 \mathrm{C}-\mathrm{C} 4-\mathrm{H} 4 \mathrm{D}$ & 108.1 & $\mathrm{C} 11 \mathrm{~A}-\mathrm{Zn} 1 \mathrm{~A}-\mathrm{Cl3A}$ & $105.66(4)$ \\
\hline $\mathrm{C} 6-\mathrm{C} 5-\mathrm{C} 4$ & $109.98(11)$ & $\mathrm{Cl} 2 \mathrm{~A}-\mathrm{Zn} 1 \mathrm{~A}-\mathrm{Cl} 3 \mathrm{~A}$ & $108.92(3)$ \\
\hline $\mathrm{C} 6-\mathrm{C} 5-\mathrm{H} 5 \mathrm{C}$ & 109.7 & $\mathrm{Cl} 3 \mathrm{~B}-\mathrm{Zn} 1 \mathrm{~B}-\mathrm{Cl} 4 \mathrm{~B}$ & $109.0(8)$ \\
\hline $\mathrm{C} 4-\mathrm{C} 5-\mathrm{H} 5 \mathrm{C}$ & 109.7 & $\mathrm{Cl} 3 \mathrm{~B}-\mathrm{Zn} 1 \mathrm{~B}-\mathrm{Cl} 2 \mathrm{~B}$ & $110.8(6)$ \\
\hline $\mathrm{C} 6-\mathrm{C} 5-\mathrm{H} 5 \mathrm{D}$ & 109.7 & $\mathrm{Cl} 14 \mathrm{~B}-\mathrm{Zn} 1 \mathrm{~B}-\mathrm{Cl} 2 \mathrm{~B}$ & $100.3(7)$ \\
\hline $\mathrm{C} 4-\mathrm{C} 5-\mathrm{H} 5 \mathrm{D}$ & 109.7 & $\mathrm{Cl} 3 \mathrm{~B}-\mathrm{Zn} 1 \mathrm{~B}-\mathrm{Cl1B}$ & $107.6(6)$ \\
\hline $\mathrm{H} 5 \mathrm{C}-\mathrm{C} 5-\mathrm{H} 5 \mathrm{D}$ & 108.2 & $\mathrm{Cl} 4 \mathrm{~B}-\mathrm{Zn} 1 \mathrm{~B}-\mathrm{Cl1B}$ & $110.7(6)$ \\
\hline $\mathrm{N} 2-\mathrm{C} 6-\mathrm{C} 1$ & $106.74(10)$ & $\mathrm{Cl} 2 \mathrm{~B}-\mathrm{Zn} 1 \mathrm{~B}-\mathrm{Cl1B}$ & $118.1(5)$ \\
\hline $\mathrm{N} 2-\mathrm{C} 6-\mathrm{C} 5$ & $113.24(10)$ & $\mathrm{O} 1 \mathrm{~W}^{\mathrm{i}}-\mathrm{C} 11 \mathrm{~B}-\mathrm{Zn} 1 \mathrm{~B}$ & $148.4(7)$ \\
\hline $\mathrm{C} 1-\mathrm{C} 6-\mathrm{C} 5$ & $111.78(10)$ & $\mathrm{C} 11 \mathrm{~B}^{\mathrm{ii}}-\mathrm{O} 1 \mathrm{~W}-\mathrm{H} 1 \mathrm{O} 1$ & $128.5(15)$ \\
\hline $\mathrm{N} 2-\mathrm{C} 6-\mathrm{H} 6$ & 108.3 & $\mathrm{C} 11 \mathrm{~B}^{\mathrm{ii}}-\mathrm{O} 1 \mathrm{~W}-\mathrm{H} 2 \mathrm{O} 1$ & $20.4(15)$ \\
\hline $\mathrm{C} 1-\mathrm{C} 6-\mathrm{H} 6$ & 108.3 & $\mathrm{H} 1 \mathrm{O} 1-\mathrm{O} 1 \mathrm{~W}-\mathrm{H} 2 \mathrm{O} 1$ & $108.4(17)$ \\
\hline $\mathrm{C} 5-\mathrm{C} 6-\mathrm{H} 6$ & 108.3 & $\mathrm{H} 1 \mathrm{O} 2-\mathrm{O} 2 \mathrm{~W}-\mathrm{H} 2 \mathrm{O} 2$ & $108.0(17)$ \\
\hline $\mathrm{N} 3-\mathrm{C} 7-\mathrm{C} 12$ & $106.99(10)$ & $\mathrm{H} 1 \mathrm{O} 3-\mathrm{O} 3 \mathrm{~W}-\mathrm{H} 2 \mathrm{O} 3$ & $105.2(17)$ \\
\hline $\mathrm{N} 3-\mathrm{C} 7-\mathrm{C} 8$ & $112.78(9)$ & & \\
\hline $\mathrm{Cr} 1-\mathrm{N} 1-\mathrm{C} 1-\mathrm{C} 6$ & $43.74(11)$ & $\mathrm{Cr} 1-\mathrm{N} 4-\mathrm{C} 12-\mathrm{C} 11$ & $165.21(8)$ \\
\hline $\mathrm{Cr} 1-\mathrm{N} 1-\mathrm{C} 1-\mathrm{C} 2$ & $166.35(9)$ & $\mathrm{N} 3-\mathrm{C} 7-\mathrm{C} 12-\mathrm{N} 4$ & $-56.47(11)$ \\
\hline $\mathrm{N} 1-\mathrm{C} 1-\mathrm{C} 2-\mathrm{C} 3$ & $-174.32(11)$ & $\mathrm{C} 8-\mathrm{C} 7-\mathrm{C} 12-\mathrm{N} 4$ & $179.35(9)$ \\
\hline $\mathrm{C} 6-\mathrm{C} 1-\mathrm{C} 2-\mathrm{C} 3$ & $-54.88(14)$ & $\mathrm{N} 3-\mathrm{C} 7-\mathrm{C} 12-\mathrm{C} 11$ & $179.60(9)$ \\
\hline $\mathrm{C} 1-\mathrm{C} 2-\mathrm{C} 3-\mathrm{C} 4$ & $56.88(15)$ & $\mathrm{C} 8-\mathrm{C} 7-\mathrm{C} 12-\mathrm{C} 11$ & $55.42(13)$ \\
\hline $\mathrm{C} 2-\mathrm{C} 3-\mathrm{C} 4-\mathrm{C} 5$ & $-58.90(14)$ & $\mathrm{C} 10-\mathrm{C} 11-\mathrm{C} 12-\mathrm{N} 4$ & $-174.49(11)$ \\
\hline $\mathrm{C} 3-\mathrm{C} 4-\mathrm{C} 5-\mathrm{C} 6$ & $57.83(14)$ & $\mathrm{C} 10-\mathrm{C} 11-\mathrm{C} 12-\mathrm{C} 7$ & $-54.61(14)$ \\
\hline $\mathrm{Cr} 1-\mathrm{N} 2-\mathrm{C} 6-\mathrm{C} 1$ & $43.07(10)$ & $\mathrm{Cr} 1-\mathrm{N} 5-\mathrm{C} 13-\mathrm{C} 18$ & $43.53(10)$ \\
\hline $\mathrm{Cr} 1-\mathrm{N} 2-\mathrm{C} 6-\mathrm{C} 5$ & $166.49(8)$ & $\mathrm{Cr} 1-\mathrm{N} 5-\mathrm{C} 13-\mathrm{C} 14$ & $166.59(9)$ \\
\hline $\mathrm{N} 1-\mathrm{C} 1-\mathrm{C} 6-\mathrm{N} 2$ & $-57.08(12)$ & $\mathrm{N} 5-\mathrm{C} 13-\mathrm{C} 14-\mathrm{C} 15$ & $-178.16(11)$ \\
\hline $\mathrm{C} 2-\mathrm{C} 1-\mathrm{C} 6-\mathrm{N} 2$ & $179.59(10)$ & $\mathrm{C} 18-\mathrm{C} 13-\mathrm{C} 14-\mathrm{C} 15$ & $-57.11(15)$ \\
\hline $\mathrm{N} 1-\mathrm{C} 1-\mathrm{C} 6-\mathrm{C} 5$ & $178.59(10)$ & $\mathrm{C} 13-\mathrm{C} 14-\mathrm{C} 15-\mathrm{C} 16$ & $56.49(16)$ \\
\hline
\end{tabular}




$\begin{array}{llll}\mathrm{C} 2-\mathrm{C} 1-\mathrm{C} 6-\mathrm{C} 5 & 55.27(13) & \mathrm{C} 14-\mathrm{C} 15-\mathrm{C} 16-\mathrm{C} 17 & -55.32(18) \\ \mathrm{C} 4-\mathrm{C} 5-\mathrm{C} 6-\mathrm{N} 2 & -176.70(11) & \mathrm{C} 15-\mathrm{C} 16-\mathrm{C} 17-\mathrm{C} 18 & 54.00(18) \\ \mathrm{C} 4-\mathrm{C} 5-\mathrm{C} 6-\mathrm{C} 1 & -56.10(14) & \mathrm{C} 1-\mathrm{N} 6-\mathrm{C} 18-\mathrm{C} 13 & 40.46(11) \\ \mathrm{Cr} 1-\mathrm{N} 3-\mathrm{C} 7-\mathrm{C} 12 & 43.41(10) & \mathrm{C} 1-\mathrm{N} 6-\mathrm{C} 18-\mathrm{C} 17 & 163.39(10) \\ \mathrm{Cr} 1-\mathrm{N} 3-\mathrm{C} 7-\mathrm{C} 8 & 167.23(8) & \mathrm{N} 5-\mathrm{C} 13-\mathrm{C} 18-\mathrm{N} 6 & -55.59(12) \\ \mathrm{N} 3-\mathrm{C} 7-\mathrm{C} 8-\mathrm{C} 9 & -176.66(11) & \mathrm{C} 14-\mathrm{C} 13-\mathrm{C} 18-\mathrm{N} 6 & -179.51(10) \\ \mathrm{C} 12-\mathrm{C} 7-\mathrm{C} 8-\mathrm{C} 9 & -55.76(14) & \mathrm{N} 5-\mathrm{C} 13-\mathrm{C} 18-\mathrm{C} 17 & -179.30(10) \\ \mathrm{C} 7-\mathrm{C} 8-\mathrm{C} 9-\mathrm{C} 10 & 56.43(15) & \mathrm{C} 14-\mathrm{C} 13-\mathrm{C} 18-\mathrm{C} 17 & 56.79(14) \\ \mathrm{C} 8-\mathrm{C} 9-\mathrm{C} 10-\mathrm{C} 11 & -57.35(15) & \mathrm{C} 16-\mathrm{C} 17-\mathrm{C} 18-\mathrm{N} 6 & -174.93(12) \\ \mathrm{C} 9-\mathrm{C} 10-\mathrm{C} 11-\mathrm{C} 12 & 55.91(15) & \mathrm{C} 16-\mathrm{C} 17-\mathrm{C} 18-\mathrm{C} 13 & -54.69(16) \\ \mathrm{C} 1-\mathrm{N} 4-\mathrm{C} 12-\mathrm{C} 7 & 42.38(10) & & \end{array}$

Symmetry codes: (i) $-x+2, y-1 / 2,-z+1 / 2$; (ii) $-x+2, y+1 / 2,-z+1 / 2$.

Hydrogen-bond geometry $\left(\AA,{ }^{\circ}\right)$

\begin{tabular}{|c|c|c|c|c|}
\hline$D-\mathrm{H} \cdots A$ & $D-\mathrm{H}$ & $\mathrm{H} \cdots A$ & $D \cdots A$ & $D-\mathrm{H} \cdots A$ \\
\hline $\mathrm{N} 1-\mathrm{H} 1 A \cdots \mathrm{Cl} 5^{\mathrm{iii}}$ & 0.91 & 2.40 & $3.2535(15)$ & 157 \\
\hline $\mathrm{N} 1-\mathrm{H} 1 B \cdots \mathrm{O} 3 W$ & 0.91 & 2.36 & $3.0178(16)$ & 129 \\
\hline $\mathrm{N} 2-\mathrm{H} 2 A \cdots \mathrm{O} 2 W$ & 0.91 & 2.01 & $2.9051(17)$ & 166 \\
\hline $\mathrm{N} 2-\mathrm{H} 2 B \cdots \mathrm{Cl} 2 A^{\mathrm{ii}}$ & 0.91 & 2.45 & $3.2197(14)$ & 142 \\
\hline $\mathrm{N} 2-\mathrm{H} 2 B \cdots \mathrm{Cl} 3 B^{\mathrm{ii}}$ & 0.91 & 2.36 & $3.180(18)$ & 150 \\
\hline $\mathrm{N} 3-\mathrm{H} 3 A \cdots \mathrm{O} 2 W$ & 0.91 & 2.13 & $2.9832(16)$ & 156 \\
\hline $\mathrm{N} 3-\mathrm{H} 3 B \cdots \mathrm{C} 11 A^{\mathrm{iv}}$ & 0.91 & 2.52 & $3.2574(13)$ & 138 \\
\hline $\mathrm{N} 3-\mathrm{H} 3 B \cdots \mathrm{Cl} 3 A^{\mathrm{iv}}$ & 0.91 & 2.77 & $3.4547(16)$ & 133 \\
\hline $\mathrm{N} 3-\mathrm{H} 3 B \cdots \mathrm{Cl} 2 B^{\mathrm{iv}}$ & 0.91 & 2.67 & $3.471(10)$ & 147 \\
\hline $\mathrm{N} 3-\mathrm{H} 3 B^{\cdots} \cdot \mathrm{Cl} 4 B^{\mathrm{iv}}$ & 0.91 & 2.68 & $3.35(2)$ & 131 \\
\hline $\mathrm{N} 4-\mathrm{H} 4 A \cdots \mathrm{Cl} 1 B^{\mathrm{v}}$ & 0.91 & 2.74 & $3.473(11)$ & 138 \\
\hline $\mathrm{N} 4-\mathrm{H} 4 B \cdots \mathrm{Cl} 2 A^{\mathrm{ii}}$ & 0.91 & 2.64 & $3.4267(15)$ & 146 \\
\hline $\mathrm{N} 4-\mathrm{H} 4 B \cdots \mathrm{O} 1 W^{\mathrm{ri}}$ & 0.91 & 2.39 & $2.9804(17)$ & 123 \\
\hline $\mathrm{N} 5-\mathrm{H} 5 A^{\cdots} \cdots \mathrm{Cl} 3 A^{\mathrm{v}}$ & 0.91 & 2.51 & $3.4245(14)$ & 178 \\
\hline $\mathrm{N} 5-\mathrm{H} 5 A^{\cdots}{ }^{-C l} 4 B^{v}$ & 0.91 & 2.73 & 3.634 (19) & 173 \\
\hline $\mathrm{N} 5-\mathrm{H} 5 B \cdots \mathrm{Cl} 1 A^{\mathrm{iv}}$ & 0.91 & 2.74 & $3.3664(16)$ & 127 \\
\hline $\mathrm{N} 5-\mathrm{H} 5 B \cdots \mathrm{O} 3 \mathrm{~W}$ & 0.91 & 2.22 & $2.9724(17)$ & 140 \\
\hline $\mathrm{N} 6-\mathrm{H} 6 A \cdots \mathrm{Cl} 5^{\mathrm{iii}}$ & 0.91 & 2.39 & $3.2474(14)$ & 158 \\
\hline $\mathrm{O} 1 W-\mathrm{H} 1 O 1 \cdots \mathrm{Cl} 5$ & $0.85(1)$ & $2.24(1)$ & $3.0878(17)$ & $179(2)$ \\
\hline $\mathrm{O} 1 W-\mathrm{H} 2 O 1 \cdots \mathrm{C} 14 A^{\mathrm{ii}}$ & $0.84(1)$ & $2.28(1)$ & $3.1170(13)$ & $174(2)$ \\
\hline $\mathrm{O} 2 W-\mathrm{H} 1 O 2 \cdots \mathrm{Cl} 1 A$ & $0.83(1)$ & $2.28(1)$ & $3.1140(12)$ & $175(2)$ \\
\hline $\mathrm{O} 2 W-\mathrm{H} 1 O 2 \cdots \mathrm{Cl} 2 B$ & $0.83(1)$ & $2.45(1)$ & $3.271(9)$ & $167(2)$ \\
\hline $\mathrm{O} 2 W-\mathrm{H} 2 O 2 \cdots \mathrm{O} 1 W$ & $0.83(1)$ & $1.92(1)$ & $2.7468(19)$ & $177(2)$ \\
\hline $\mathrm{O} 3 W-\mathrm{H} 1 O 3 \cdots \mathrm{Cl} 5^{\text {iv }}$ & $0.84(1)$ & $2.41(1)$ & $3.2139(13)$ & $159(2)$ \\
\hline $\mathrm{O} 3 W-\mathrm{H} 2 O 3 \cdots \mathrm{Cl} 2 A^{\mathrm{iv}}$ & $0.84(1)$ & $2.38(1)$ & $3.2153(17)$ & $175(2)$ \\
\hline $\mathrm{O} 3 W-\mathrm{H} 2 O 3 \cdots \mathrm{Cl} 3 B^{\text {iv }}$ & $0.84(1)$ & $2.23(2)$ & $3.05(2)$ & $167(2)$ \\
\hline
\end{tabular}

Symmetry codes: (ii) $-x+2, y+1 / 2,-z+1 / 2$; (iii) $x,-y+3 / 2, z+1 / 2$; (iv) $-x+1, y+1 / 2,-z+1 / 2$; (v) $x, y+1, z$. 